\title{
Predictive Significance of VEGF and HIF-1 $\alpha$ Expression in Patients with Metastatic Colorectal Cancer Receiving Chemotherapy Combinations with Bevacizumab
}

\author{
Veli Berk ${ }^{1 *}$, Kemal Deniz ${ }^{2}$, Oktay Bozkurt ${ }^{1}$, Ersin Ozaslan$^{1}$, Halit Karaca ${ }^{1}$, \\ Mevlude Inanc ${ }^{1}$, Ayse Ocak Duran' ${ }^{1}$, Metin Ozkan ${ }^{1}$
}

\begin{abstract}
Background: There is no suggested molecular indicator for the determination of which patients will benefit from anti-angiogenetic treatment in metastatic colorectal cancers. Materials and Methods: In this study, VEGF and HIF-1 $\alpha$ expression and their clinical significance were studied in tumor tissues of patients with colorectal cancer receiving bevacizumab-based treatment. VEGF and HIF-1 $\alpha$ were assessed by immunohistochemistry in the primary tumors of $\mathbf{5 3}$ metastatic colorectal cancer patients receiving chemotherapy in combination with first line bevacizumab. Results: The clinical benefit rate in the low-VEGF expression group was 38\%, while it was $62 \%$ in the high expression group. While the median progression-free survival (PFS) was 10 months in the high-VEGF expression group, it was 8 months in the low-VEGF expression group $(p=0.009)$. The median overall survival (OS) was found to be 26 months vs 15 months. Thus, when VEGF was strongly expressed it was in favor of that group and the difference was statistically significant $(p=0.03)$. High VEGF expression rate was an independent factor that correlated with OS or PFS ( $p=0.016$ and 0.009 , respectively). Conclusions: The data showed that VEGF may have predictive value for determining the treatment of CRC.
\end{abstract}

Keywords: Colorectal cancer - VEGF - HIF-1 $\alpha$ - Bevacizumab

Asian Pac J Cancer Prev, 16 (14), 6149-6154

\section{Introduction}

With better understanding of the developmental processes at the molecular level, targeted therapies have been introduced in the treatment of metastatic colorectal cancer. With the introduction of targeted therapies developed against Vascular Endothelial Growth Factor (VEGFR) and Epidermal Growth Factor (EGFR) receptors since 2004, survival in metastatic colorectal cancer has been prolonged to 30 months.

Observation of angiogenesis around tumor cells dates back for many years (Goldman, 1907). VEGF increases vascular permeability and is known as the most potent angiogenic protein. Tumor development and metastatic property depend on the formation of new vessels (Folkman, 1995). At first, permeability in capillaries around the tumor is increased and the passage of plasma proteins in the circulation is allowed. This step is essential for the onset of angiogenesis (Brown et al.,1997). At the same time, the fibrin present in the extracellular space acts as a skeleton for the migrating endothelial cells. Potential for metastasis occurs as a result of the entrance of tumor cells into the blood flow (Kajdaniuk et al., 2011). Due to VEGF, endothelial cells proliferate and allow tube formation as preliminaries for new vessels by migrating and aligning. VEGF-A is known as human VEGF. The VEGF mentioned in many known studies in the literature is VEGF-A. VEGF molecules act on different receptors (Wollenhaupt et al., 2004). VEGF has been shown to have a prognostic significance in many malignancies: predominantly breast, colon and renal cancers (Zhao et al., 2004, Saad et al., 2004, Liang et al., 2009). In colon cancer, VEGF expression was increased in invasive tumors compared with premalignant lesions (Wong et al.,1999). Overexpressed VEGF in primary tumor tissue is one of the most important pathways for the metastasis of tumor cells. In metastatic colorectal cancers, both VEGF and VEGF receptors are known to be highly expressed. VEGF expressions were found to be weaker in non-metastatic colorectal cancers compared with metastatic cases (De Vita et al., 2004). Strength of VEGF expression is associated with poor prognosis (Liang et al., 2010). Recently, anti-angiogenesis has been used as a novel treatment alternative. In particular, stimulation of angiogenesis by VEGF has prompted the use of VEGF as the target. Overexpressed VEGF in tumor tissue is mostly found in the vessels feeding the tumor (Dvorak et al.,1991). Systemic administration of the VEGF antibody will lead to

${ }^{1}$ Department of Medical Oncology, ${ }^{2}$ Department of Pathology, Erciyes University Medical Faculty, Kayseri, Turkey *For correspondence: veliberk@gmail.com 
accumulation within the tumor vessels in high specificity. Consequently, tumor growth will stop. Bevacizumab is a recombinant humanized monoclonal antibody developed against VEGF. Its addition to chemotherapy is known to be useful, particularly in advanced stage colorectal cancers (Kabbinavar et al., 2003). Although bevacizumab provides significant benefit in the clinical treatment of metastatic colorectal cancer, this does not differ in K-RAS mutant or wild types (Hurwitz et al., 2009).Currently, a molecular marker or tumor characteristic to determine those patients who may benefit from anti-VEGF therapy in the treatment of metastatic colorectal cancer has not been found (Mahfud et al., 2010).

Hypoxia-inducible factor 1 (HIF-1) is a heterodimeric transcription factor meeting the oxygen need arising during tumor growth. The main activity is carried out by the HIF$1 \alpha$ subunit. Furthermore, activation of HIF- $1 \alpha$ that is independent of oxygen, as a result of genetic changes also contributes to tumor growth and development (Mabjeesh et al., 2007). It is thought that overexpression of HIF-1 $\alpha$ causes increased angiogenesis and consequently tumor growth, metastasis and also resistance to chemotherapy and radiotherapy. Overexpressed HIF-1 $\alpha$ was found to be a poor prognostic factor primarily in renal cell cancer, colorectal cancer and in many other diseases (Dorević et al., 2009, Baba et al., 2010, Dungwa et al., 2012). Many pathways are activated as a result of HIF stimulation and VEGF is the leading one among these (Bertout et al., 2008). These studies have shown that HIF and associated pathways can be potential treatment targets in the treatment of many cancers.

Recently, personalized treatment has become popular in the treatment of many cancers. With targeted therapies, these patients may have a better prognosis when molecules causing poor prognosis are targeted. The aim of this study is to evaluate the predictive value of VEGF and HIF- $1 \alpha$ expression intensities in the determination of anti-angiogenic therapy in patients with colorectal cancer.

\section{Materials and Methods}

Patients presenting at the Erciyes University Medical Faculty Hospital Medical Oncology Outpatient Clinic with the diagnosis of metastatic colorectal cancer confirmed with histopathology between April 2004 and October 2011 were included in the study. A total of 53 (24 female and 29 male) patients receiving bevacizumab added to first line chemotherapy during treatment were included in the study. The mean age was 55 (range, 32-79 years). Consent was obtained from the Local Ethics Committee before the study (Consent number: 2012/12). Patient charts were retrospectively screened. All patients had physical examinations, chest, abdomen and pelvis computerized tomography carried out at onset for the purpose of staging. Whole body bone scintigraphy was performed when necessary. Age, gender, diagnosis dates, histopathologic diagnosis, clinicopathological data at diagnosis and metastatic status, chemotherapy regimens, adverse effects, progression free survival (PFS) and overall survival (OS) durations, treatment responses and final control data or exitus dates of the patients were evaluated. Bevacizumab was added to the chemotherapy combinations of FOLFIRI and XELOX (capecitabine, oxaliplatin) protocols. Patients were evaluated every 2-3 months with physical examination and computed tomography and laboratory tests. Evaluation was classified according to Response Evaluation Criteria in Solid Tumors (RECIST). Complete response was regarded as absence of detectable disease and absence of disease related symptoms for 4 weeks. Partial response was accepted as $50 \%$ or more decrease in the greatest diameter of the targeted lesion and absence of a new lesion within 4 weeks. Stable disease was defined as absence of a $25 \%$ decrease or increase or no change in the size of the tumor; progressive disease was defined as appearance of a new lesion or more than $25 \%$ increase in the size of the tumor. Clinical benefit was defined as the sum of all responses persisting over 4 weeks and stable disease.

Sections $4 \mu \mathrm{m}$ thick from the formalin fixed paraffin blocks of primary tumors of the patients in the archives of Erciyes University Medical Faculty Department of Pathology were transferred to poly-l-lysine coated slides in order to evaluate VEGF and HIF-1 $\alpha$ overexpression. Tissue sections were deparaffinized with xylol and dehydrated with alcohol. VEGF primary antibody (Thermo scientific Ab-1, U.K) was incubated at 1:25 dilution and HIF- $1 \alpha$ primary antibody (Thermo scientific Ab-4, (Clone H1 alpha 67, U.K) at 1:50 dilution for 30 minutes during which immunohistochemical staining was carried out with Ventana Benchmark Autostainer.

During evaluation, cell counts were carried out at 400x magnification by counting 200 cells in each area of at least 5 randomly selected areas. Expression of VEGF and HIF-1 $\alpha$ was detected with the observation of immune reactivity in the cytoplasm of tumor cells. To assess staining diffusion, 5 different subscores were defined depending on the rate of tumor cells stained positively; 0 (below 5\%), 1 (6\%-25\%), 2 (26\%-50\%), 3 (51\%-75\%), 4 (above $75 \%$ ). Staining intensity was scored between 0 and 3: 0 (negative), 1 (mildly positive), 2 (moderately positive) and 3 (strongly positive). The final score was obtained by multiplying intensity and diffusion scores; 0 (negative), + (1-4), ++ (5-8) and +++ (Dvorak et al.,1991, Wong et al.,1999, De Vita et al., 2004, Liang et al., 2010). For statistical evaluation, those with final scores of 0 or + were classified as the weakly expressed group and those with scores ++ and +++ as the strongly expressed group.

Data were evaluated using the SPSS v 15.0 program. When calculating OS, date of death or date of final control was taken from date of treatment onset. On the other hand, when calculating PFS, date of detection of any clinical or radiologic progression on the first day of treatment was taken. The Kaplan-Meier method was used for calculating survival according to the HIF- $1 \alpha$ and VEGF expression scores of the patients and log-rank test was used for comparison. Chi-squared test and two-sided Fisher exact test were used for comparing the treatment response rates of the two groups separately regarding staining characteristics. Multivariate analysis were done with a Cox regression model. A p value $<0.05$ was regarded as statistically significant. 


\section{Results}

A total of 53 patients with metastatic colorectal cancer were evaluated. The clinicopathological data of the patients are shown in the table (Table 1).

VEGF was highly expressed in $30(57 \%)$ patients and there was low VEGF expression in $23(43 \%)$ patients. HIF-1 $\alpha$ was highly expressed in $29(55 \%)$ patients and lowly expressed in $24(45 \%)$ patients. In patients receiving first line combined chemotherapy with bevacizumab, $5(9 \%)$ had progression and $48(91 \%)$ showed clinical benefit. When VEGF expression was compared with treatment response rates, the rate of clinical benefit was $38 \%$ in the weakly expressed group, while this rate was $62 \%$ in the highly expressed group. This difference was statistically significant $(\mathrm{p}=0.01)$. All the patients who had progression in the evaluation of response to first line chemotherapy had low VEGF expressions. There was no significance between HIF-1 $\alpha$ expression and treatment response rates. While PFS was 10 months in the group with strong VEGF expression, it was 8 months in the group with low expression and this difference was statistically significant $(\mathrm{p}=0.009)$ (Figure 1). Evaluation regarding OS, was 26 months versus 15 months in favor of the group with strong VEGF expression and this difference was statistically significant $(\mathrm{p}=0.03)$ (Figure 2$)$. There was no difference between groups with low and high HIF-1 $\alpha$ expression regarding treatment response rates. Clinical benefit rate was $48 \%$ in the group with low HIF$1 \alpha$ expression and $52 \%$ in the group with strong HIF- $1 \alpha$ expression. While PFS was 10 months in the group with highly expressed HIF-1 $\alpha$, it was 9 months in the group with low expression. The difference between PFS was not Table 1. Clinical and Histopathological Characteristics of the Patients

\begin{tabular}{lc}
\hline & Patient $(\mathrm{n}=53) \mathrm{n}(\%)$ \\
\hline Age (median) years & 55 (Range, 32-79) \\
Gender & $29(55)$ \\
$\quad$ Male & $24(45)$ \\
Female & \\
Histopathology & $49(93)$ \\
$\quad$ Adenocarcinoma & $4(7)$ \\
Mucinous adenocarcinoma & \\
Metastasis Region Number & $41(77)$ \\
One & $12(23)$ \\
Numerous & \\
Chemotherapy & $38(72)$ \\
FOLFIRİ & $15(28)$ \\
XELOX & \\
Performance status (ECOG) & $29(54)$ \\
0 & $20(38)$ \\
1 & $4(8)$ \\
2 & \\
Localization & $18(34)$ \\
Rectum & $18(34)$ \\
Sigmoid & $17(32)$ \\
Colon & \\
Stage of first diagnosis & $37(70)$ \\
IV & $15(28)$ \\
III & $1(2)$ \\
II & \\
\hline
\end{tabular}

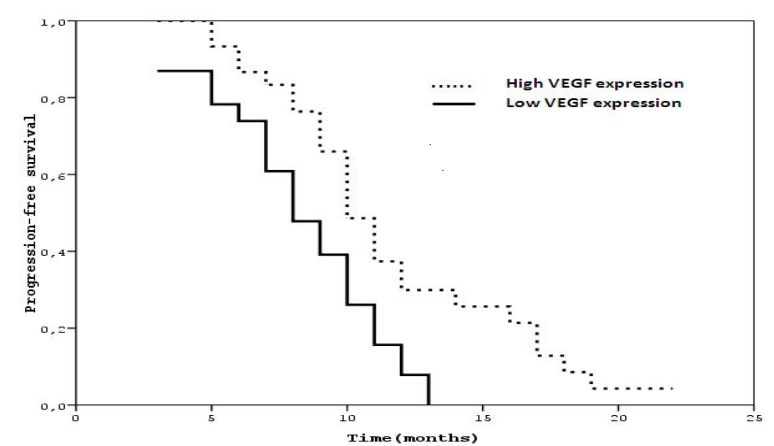

Figure 1. VEGF Expression and Progression free Survival in Patients Receiving First Line Combined Therapy with Bevacizumab $(p=0.009)$

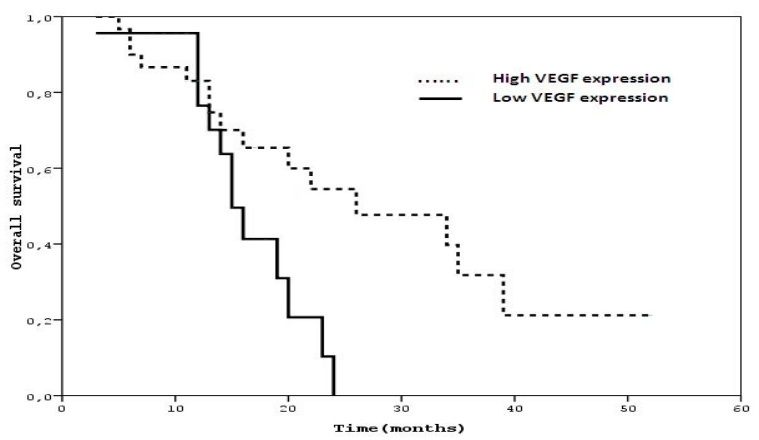

Figure 2. VEGF Expression and Overall Survival in Patients Receiving First Line Combined Therapy with Bevacizumab $(p=0.03)$

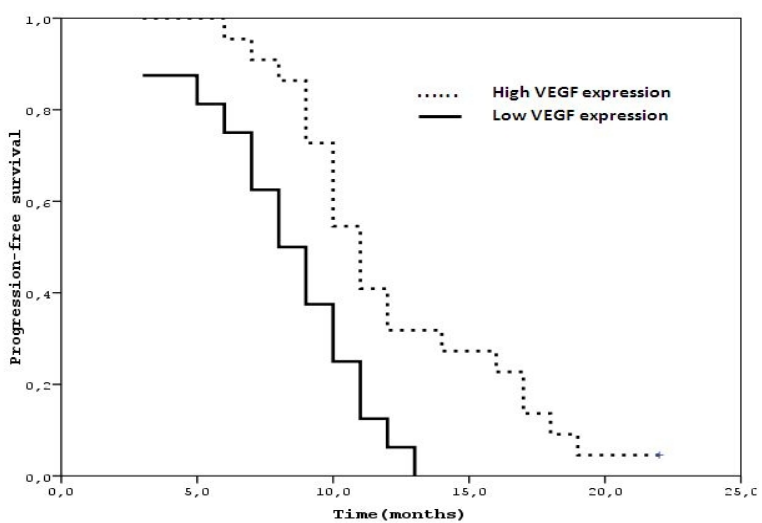

Figure 3. Progression Free Survival and VEGF Expression in Patients Undergoing First Line FOLFIRI-Bevacizumab Combination $(p=0.009)$

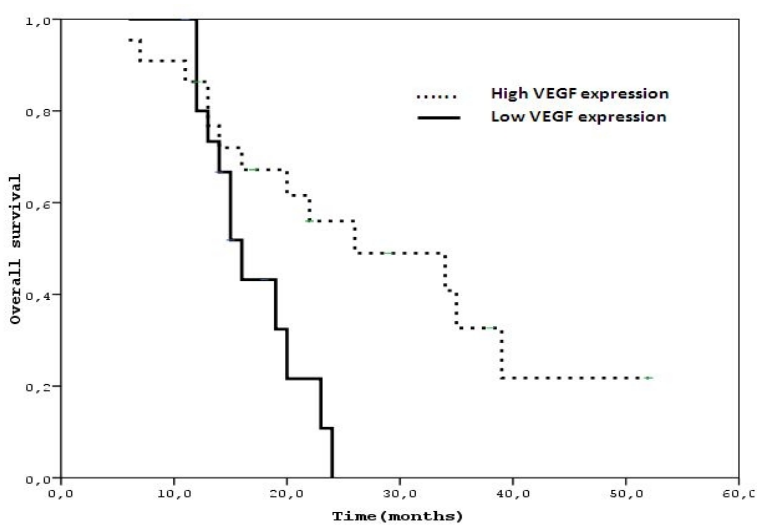

Figure 4. Overall Survival and VEGF Expression in Patients Undergoing First Line FOLFIRI-Bevacizumab Combination $(\mathbf{p}=\mathbf{0 . 0 4})$ 
Table 2. Multivariate Prognostic Factors for Progression free Survival (PFS) and Overall Survival (OS)

\begin{tabular}{|c|c|c|c|c|c|c|}
\hline \multirow[t]{2}{*}{ Variables } & \multicolumn{3}{|c|}{ PFS } & \multicolumn{3}{|c|}{ OS } \\
\hline & Multivaria & te HR $(95 \%$ CI $)$ & $\mathrm{p}$ & Multivaria & e HR $(95 \% \mathrm{CI})$ & $\mathrm{p}$ \\
\hline Age (years) & 1.03 & $(0.90-1.04)$ & 0.31 & 1.09 & $(1.03-1.117)$ & 0.16 \\
\hline Gender (male/female) & 1.17 & $(0.21-3.36)$ & 0.81 & 15.79 & $(1.12-222.26)$ & 0.041 \\
\hline K-ras status (wild/mutant type) & 9.90 & $(1.87-52.4)$ & 0.07 & 16.21 & $(6.1-43.09)$ & 0.002 \\
\hline Dose reduction (no/yes) & 4.34 & $(1.32-25.0)$ & 0.1 & 8.33 & $(1.31-52.6)$ & 0.024 \\
\hline Dose delay (no/yes) & 1.009 & $(0.22-4.52)$ & 0.99 & 1.40 & $(0.17-30.83)$ & 0.714 \\
\hline ECOG PS (PS: 0-1/2) & 2.53 & $(0.019-52.6)$ & 0.53 & 4.71 & $(0.11-16.84)$ & 0.71 \\
\hline Metastases (single/multipl organ) & 5.31 & $(1.59-17.85)$ & 0.055 & 22.22 & $(1.44-33.3)$ & 0.026 \\
\hline CT regimen (irinotecan/oxaliplatin-based) & 7.14 & $(1.28-40.0)$ & 0.025 & 8.77 & $(0.10-45.61)$ & 0.478 \\
\hline HIF status(high/low) & 2.11 & $(0.64-6.92)$ & 0.21 & 3.49 & $(0.02-47.74)$ & 0.618 \\
\hline VEGF status(high/low) & 20.0 & $(1.76-250)$ & 0.016 & 100.0 & $(5.52-1000.0)$ & 0.009 \\
\hline
\end{tabular}

statistically significant. OS in both groups was 20 months.

When VEGF and HIF-1 $\alpha$ expressions in the primary tumors of the patients with and without metastasis at diagnosis were compared, the groups were similar. There were no differences between VEGF and HIF- $1 \alpha$ expression rates in patients with single site metastasis and multiple site metastases. When the association between efficiency of chemotherapy protocols combined with bevacizumab and VEGF and HIF- $1 \alpha$ expressions were analyzed,PFS was 11 months with FOLFIRI and 8 months with XELOX in the group with overexpressed VEGF, while it was 8 months with both protocols in the group with low expression. PFS with FOLFIRI-Bevacizumab combination was different from other groups in the group with high VEGF expression and this difference was statistically significant $(\mathrm{p}=0.009)$ (Figure 3). When OS was evaluated, it was 26 months in the group with high VEGF expression and 16 months in the group with low expression with FOLFIRI ( $p=0.04$ ) (Figure 4). Median OS was not reached in the group receiving XELOX. There were no significant survival durations between the HIF-1 $\alpha$ expression rates and the chemotherapy regimens applied. When treatment responses were analyzed, the clinical benefit rate was $58 \%$ in the group with high VEGF expression and $34 \%$ in those with low expression in 38 patients undergoing FOLFIRI regimen. Although this difference was not statistically significant, it approached the limit of significance $(\mathrm{p}=0.06)$. In this group of patients, all 3 patients whose treatment responses were in progression were in the group with low VEGF expression. On the other hand, no association was noted between VEGF expression and treatment responses in 15 patients receiving XELOX. No relationship was found between the efficiency of treatment regimens and HIF-1 $\alpha$ expression. No association was found between post-treatment adverse effects such as neutropenia, mucositis, diarrhea and VEGF and HIF-1 $\alpha$ expression.

In multivariate analysis, high VEGF expression rate was an independent factor that correlated with PFS and OS ( $\mathrm{p}=0.016$ and 0.009 , respectively) (Table-2).

\section{Discussion}

VEGF and HIF-1 $\alpha$ are known to be responsible for tumor angiogenesis and progression in colorectal cancer as in some other tumors. Overexpression of VEGF and
HIF-1 $\alpha$ has been shown to be an independent prognostic factor and to affect survival (Bertout et al., 2008, Dorević et al., 2009, Okita et al., 2009, Baba et al., 2010, Dungwa et al., 2012). K-RAS mutation in the EGFR pathway is a predictive factor for personalization of treatment in metastatic colorectal cancer. However, an indicator to determine the treatment specific for the patient in the VEGF pathway has not been established so far. In this study, the predictive significance of VEGF and HIF-1 $\alpha$ with demonstrated prognostic efficiency was evaluated in the treatment of metastatic colorectal cancer. Bevacizumab is lowers interstitial fluid pressure by decreasing the diameter, density and permeability of tumor vessels and increases tumor perfusion. Consequently, the efficacy of chemotherapy is enhanced. In this study it was seen that patients with overexpressed VEGF in their tumor cells had greater benefit from anti-VEGF therapy than those with low expression. The role of the degree of HIF-1 $\alpha$ expression on determining anti-VEGF therapy has not been shown. These results showed that overexpression of VEGF significantly increased both treatment response and survival with chemotherapy including bevacizumab in patients with metastatic colorectal cancer.

In a study evaluating VEGF and HIF-1 immunohistochemically in normal colon mucosa, both parameters were negative. In the same study, the overexpression rate was found to be $65 \%$ for VEGF and $44 \%$ for HIF- $1 \alpha$ in tissues with colon cancer (Wu et al., 2010). In this study, the rate of high VEGF expression was found to be $57 \%$ and overexpression of HIF- $1 \alpha$ to be $55 \%$ in tissues with metastatic colorectal cancer.

Bevacizumab was shown to be well-tolerated by patients in the initial clinical studies (Gordon et al., 2001). In the phase 2 randomized study by Kabbinavar et al (Kabbinavar et al., 2003). 5-FU/LV and bevacizumab were administered to patients with metastatic colorectal cancer. Those progressing in the placebo arm of the study were cross-shifted to the bevacizumab arm. PFS, OS and response rates were better in the 5-FU/LV and bevacizumab arm than in the single chemotherapy arm. Hurwitz et al. compared first line bolus 5-FU/LV and irinotecan added to bevacizumab with 5-FU/LV and irinotecan alone in patients with metastatic colorectal cancer. Also in that study, survival and treatment response rates were significantly increased with the bevacizumab combination than in the placebo group (Hurwitz et 
al., 2004). A study on the addition of bevacizumab to oxaliplatin based chemotherapy was carried out by Saltz et al (Saltz et al., 2008). 4 years after these studies. Bevacizumab was added to both FOLFOX4 and XELOX protocols and compared with placebo. Regarding PFS, the addition of bevacizumab to those receiving oxaliplatin based therapy provided statistical significance. However, although OS data were better than the placebo group, it did not reach statistical significance. Response rates did not change with the addition of bevacizumab. In the following BEAT and BRITE observational studies, it was shown that endpoint results were significantly improved and adverse effect profile was tolerable with the addition of bevacizumab to the treatment of metastatic colorectal cancer (Kozloff et al., 2009,Van Cutsem et al., 2009). As it seen from these studies, survival and treatment efficacy were improved with the addition of bevacizumab to 5-FU, irinotecan and oxaliplatin. However, which patients benefited from this treatment was not demonstrated in any of the studies.

In a study by Cao D et al (Cao D et al., 2009). the VEGF and HIF- $1 \alpha$ expressions of patients with operated colorectal cancer were studied regarding survival according to intensity. At the end of 5-year follow-up, overall survival was significantly less in both groups with overexpressed VEGF and HIF-1 $\alpha$ than in those with low expression. This and similar studies provided guidance in the determination of treatment targets in patients with colorectal cancer. In another study, a predictive value of VEGF expression levels was not found in patients receiving IFL combination with bevacizumab (Jubb et al., 2006). On the other hand, in a study carried out in metastatic small cell lung cancer, treatment response rates were higher with the addition of bevacizumab in patients with high plasma VEGF levels (Dowlati et al., 2008). In a similar study, those with high VEGF expression benefited more from bevacizumab therapy in breast cancer (Yang et al., 2008). In a recent review by Watanabe T et al. a model was formed for determining bevacizumab response with the RT-PCR method. VEGF, TYMS, and TIMP-3 expression levels were found to be significant in determining bevacizumab response. However, a difference was not found between FOLFIRI and FOLFOX6 regarding treatment response (Watanabe et al., 2011). In our study, while the clinical benefit rate was $62 \%$ in patients with high VEGF expression, it was $32 \%$ in those with low expression. As a reflection of this, PFS was 10 months in the group with strong expression and 8 months in the group with weak expression. Regarding OS, it was 26 months versus 15 months, respectively, but none of these differences were statistically significant. There were no differences between treatment responses and survival with HIF- $1 \alpha$. This study showed that bevacizumab seems to be more efficient in patients with high VEGF expression. Confirmation of these results with larger patient groups is important regarding the personalization of anti-VEGF therapy.

There were no differences in outcomes with consecutive administrations of irinotecan and oxaliplatin in the first and second line therapy of metastatic colorectal cancer(Tournigand et al., 2004, Hebbar et al., 2006). It is known that both agents have the same efficiency in first line therapy. Current guidelines recommend the addition of anti-VEGF therapy for both agents in first line treatment. In addition to this, in the study by Del Rio $\mathrm{M}$ et al. it was found that 14 genes play a role in determining FOLFIRI response in patients with metastatic colorectal cancer and the expressions of the 14 genes were elevated in those responding to FOLFIRI combination. Most of these 14 genes were reported to be responsible for adhesion and vascularization processes (Del Rio et al., 2007). In the phase 2 study administering first line FOLFIRIBevacizumab, PFS was 12.8 months, OS was 31.3 months and response rate was $65 \%$ (Kopetz et al., 2010). In the combined study with XELOX-Bevacizumab, PFS was 11 months, OS was 27.4 months and response rate was $72 \%$. In our study, both PFS and OS were significantly better in the FOLFIRI-Bevacizumab arm compared with the XELOX-Bevacizumab arm in patients with high VEGF expression. It would be suitable to re-evaluate these results in similar studies with similar patient numbers. Survival and treatment response rates were not found to be different among different chemotherapeutic agents in those with low VEGF expression. Again similarly, no predictive value was found in HIF-1 $\alpha$ expression for determining treatment. In our study, although FOLFIRIBevacizumab appeared to be better in the patient group with high VEGF expression, the patient number in the XELOX-Bevacizumab arm was small and randomized studies are needed to reach such a conclusion.

Patients with a high potential for adverse effects during anti-angiogenic therapy should be evaluated before treatment regarding this aspect. Combinations containing bevacizumab should not be given to patients with colon diverticulum with increased risk for perforation or bleeding tendencies. Bevacizumab use should be limited in patients with a history of hypertension, proteinuria and thromboembolism. Furthermore, because administration of bevacizumab to patients who will not benefit may involve cost increase, it is important to determine which patient will receive which agent.

It is emphasized in this study that anti-VEGF therapy should be given priority particularly in patients with high VEGF expression in determining first line therapy in the treatment of metastatic colorectal cancer.

\section{References}

Baba Y, Nosho K, Shima K, et al (2010). HIF1A overexpression is associated with poor prognosis in a cohort of 731 colorectal cancers. Am J Pathol, 176, 2292-301.

Bertout JA, Patel SA, Simon MC(2008). The impact of O2 availability on human cancer. Nat Rev Cancer, 8, 967-75.

Brown LF, Detmar M, Claffey K, et al (1997). Vascular permeability factor/vascular endothelial growth factor: a multifunctional angiogenic cytokine. EXS, 79, 233-69.

Cao D, Hou M, Guan YS, Jiang M, Yang Y, Gou HF(2009). Expression of HIF-1alpha and VEGF in colorectal cancer: association with clinical outcomes and prognostic implications. BMC Cancer, 9, 432.

Del Rio M, Molina F, Bascoul-Mollevi C, et al (2007). Gene expression signature in advanced colorectal cancer patients select drugs and response for the use of leucovorin, 
fluorouracil, and irinotecan. J Clin Oncol, 25, 773-80.

De Vita F, Orditura M, Lieto E, et al (2004). Elevated perioperative serum vascular endothelial growth factor levels in patients with colon carcinoma. Cancer, 100, 270-78.

Dorević G, Matusan-Ilijas K, Babarović E, et al (2009). Hypoxia inducible factor-1alpha correlates with vascular endothelial growth factor $\mathrm{A}$ and $\mathrm{C}$ indicating worse prognosis in clear cell renal cell carcinoma. J Exp Clin Cancer Res, 20, 28- 40.

Dowlati A, Gray R, Sandler AB, Schiller JH, Johnson DH(2008). Cell adhesion molecules, vascular endothelial growth factor, and basic fibroblast growth factor in patients with non-small cell lung cancer treated with chemotherapy with or without bevacizumab-an Eastern Cooperative Oncology Group Study. Clin Cancer Res, 14, 1407-12.

Dungwa JV, Hunt LP, Ramani $\mathrm{P}(2012)$. HIF-1 $\alpha$ up-regulation is associated with adverse clinicopathological and biological factors in neuroblastomas. Histopathology, 61, 417-27.

Dvorak HF, Sioussat TM, Brown LF, et al (1991). Distribution of vascular permeability factor (vascular endothelial growth factor) in tumors: concentration in tumor blood vessels. $J$ Exp Med, 174, 1275-8.

Folkman J(1995). Angiogenesis in cancer, vascular, rheumatoid and other disease. Nat Med, 1, 27-31.

Goldman E(1907). The growth of malignant disease in man and the lower animals with special reference to the vasculer system. Lancet, 2, 1236-40.

Gordon MS, Margolin K, Talpaz M, et al (2001). Phase I safety and pharmacokinetic study of recombinant human antivascular endothelial growth factor in patients with advanced cancer. J Clin Oncol, 19, 843-50.

Hebbar M, Tournigand C, Lledo G, et al (2006). Oncology Multidisciplinary Research Group (GERCOR). Phase II trial alternating FOLFOX-6 and FOLFIRI regimens in secondline therapy of patients with metastatic colorectal cancer (FIREFOX study). Cancer Invest, 24, 154-9.

Hurwitz H, Fehrenbacher L, Novotny W, et al (2004). Bevacizumab plus irinotecan, fluorouracil, and leucovorin for metastatic colorectal cancer. N Engl J Med, 350, 2335-42.

Hurwitz HI, Yi J, Ince W, Novotny WF, Rosen O (2009). The clinical benefit of bevacizumab in metastatic colorectal cancer is independent of K-ras mutation status: analysis of a phase III study of bevacizumab with chemotherapy in previously untreated metastatic colorectal cancer. Oncologist, 14, 22-8.

Jubb AM, Hurwitz HI, Bai W, et al (2006). Impact of vascularendothelial growth factor-A expression, thrombospondin-2 expression, and microvessel density on the treatment effect of bevacizumab in metastatic colorectal cancer. J Clin Oncol, 24, 217-27.

Kabbinavar F, Hurwitz HI, Fehrenbacher L, et al (2003). Phase II, randomized trial comparing bevacizumab plus fluorouracil (FU)/leucovorin (LV) with FU/LV alone in patients with metastatic colorectal cancer. J Clin Oncol, 21, 60-65.

Kajdaniuk D, Marek B, Foltyn W, Kos-Kudła B(2011). Vascular endothelial growth factor (VEGF) - part 2: in endocrinology and oncology. Endokrynol Pol, 62, 456-64.

Kopetz S, Hoff PM, Morris JS, et al (2010). Phase II trial of infusional fluorouracil, irinotecan, and bevacizumab for metastatic colorectal cancer: efficacy and circulating angiogenic biomarkers associated with therapeutic resistance. J Clin Oncol, 28, 453-9.

Kozloff M, Yood MU, Berlin J, et al; Investigators of the BRiTE study(2009). Clinical outcomes associated with bevacizumab-containing treatment of metastatic colorectal cancer:the BRiTE observational cohort study. Oncologist, 14, 862-70.

Liang JF, Wang HK, Xiao H, et al (2010). Relationship and prognostic significance of SPARC and VEGF protein expression in colon cancer. J Exp Clin Cancer Res, 29, 71.

Liang YX, He HC, Han ZD, et al (2009). CD147 and VEGF expression in advanced renal cell carcinoma and theirprognostic value. Cancer Invest, 27, 788-93.

Mabjeesh NJ, Amir S(2007). Hypoxia-inducible factor (HIF) in human tumorigenesis. Histol Histopathol, 22, 559-72.

Mahfud M, Breitenstein S, El-Badry AM, et al (2010). Impact of preoperative bevacizumab on complications after resection of colorectal liver metastases: case-matched control study. World J Surg, 34, 92-100.

Okita NT, Yamada Y, Takahari D, et al (2009). Vascular endothelial growth factor receptor expression as a prognostic marker for survival in colorectal cancer. Jpn J Clin Oncol, 39, 595-600.

Saad RS, Liu YL, Nathan G, Celebrezze J, Medich D, et al (2004). Endoglin (CD105) and vascular endothelial growth factor as prognostic markers in colorectal cancer. Mod Pathol, 17, 197-203.

Saltz LB, Clarke S, Díaz-Rubio E, et al (2008). Bevacizumab in combination with oxaliplatin-based chemotherapy as firstline therapy in metastatic colorectal cancer: a randomized phase III study. J Clin Oncol, 26, 2013-9.

Tournigand C, André T, Achille E, et al (2004). FOLFIRI followed by FOLFOX6 or the reverse sequence in advanced colorectal cancer: a randomized GERCOR study. J Clin Oncol, 22, 229-37.

Van Cutsem E, Rivera F, Berry S, et al (2009). Safety and efficacy of first-line bevacizumab with FOLFOX,XELOX, FOLFIRI and fluoropyrimidines in metastatic colorectal cancer: the BEAT study. Ann Oncol, 20, 1842-7.

Watanabe T,Kobunai T, Yamamoto Y, et al (2011).Gene expression of vascular endothelial growth factor A, thymidylate synthase, and tissue inhibitor of metalloproteinase 3 in prediction of response to bevacizumab treatment in colorectal cancer patients. Dis Colon Rectum, 54, 1026-35.

Wollenhaupt K, Welter H, Einspanier R, Manabe N, Brussow $\mathrm{KP}$ (2004). Expression of epidermal growth factor receptor (EGF-R), vascular endothelial growth factor receptor (VEGF-R) and fibroblast growth factor receptor (FGF-R) syst ems in porcine oviduct and endometrium during the time of implantation. J Reprod Dev, 50, 269-78.

Wong MP, Cheung N, Yuen ST, Leung SY, Chung LP(1999). Vascular endothelial growth factor is up-regulated in the early pre-malignant stage of colorectal tumour progression. Int J Cancer, 81, 845-50.

Wu Y, Jin M, Xu H, et al (2010). Clinicopathologic significance of HIF-1 $\alpha$, CXCR4, and VEGF expression in colon cancer. Clin Dev Immunol, 2010. doi:pii: 537531.

Yang SX, Steinberg SM, Nguyen D, et al (2008). Gene expression profile and angiogenic marker correlates with response to neoadjuvant bevacizumab followed by bevacizumab plus chemotherapy in breast cancer. Clin Cancer Res, 14, 5893-9.

Zhao J, Yan F, Ju H, Tang J, Qın J (2004). Correlation between serum vascular endothelial growth factor and endostatin levels in patients with breast cancer. Cancer Lett, 204, 87-95. 